\title{
Conveying Three-Dimensional Shape with Texture
}

\author{
Sunghee Kim* \\ University of Minnesota
}

\author{
Haleh Hagh-Shenas ${ }^{\dagger}$ \\ University of Minnesota
}

\author{
Victoria Interrante \\ University of Minnesota
}

\begin{abstract}
Studies have shown that although shading can give an image a "natural" look and is an important shape cue, visual perception of surface shape from shading only is severely limited when the surface is viewed locally without other visual cues such as occluding contours [Mamassian and Kersten 1996; Erens et al. 1993]. Research has shown that when the "right" texture is added to the surface, observers can reliably infer the 3D structure of the underlying shape. In our own previous work, we have found that the performance of subjects' shape judgment is significantly better with principal direction oriented texture pattern than other directional texture following either a uniformly constant direction or varying non-geodesic paths unrelated to the surface geometry. In this paper, we report our findings of a new study further investigating the effect of anisotrpic textures on shape perception when the surface texture is represented in the form of a pattern of luminance variations as well as of surface relief variations. We predicted that 1) performance would be higher with relief textures than luminance textures, and that 2) the performance would be poorer with the anisotropic textures that do not follow principal directions. The results confirmed both of our predictions.
\end{abstract}

CR Categories: I.3.7 [Computer Graphics]: Three-Dimensional Graphics and Realism-Color, shading, shadowing, and texture; I.4.8 [Image Processing and Computer Vision]: Scene AnalysisShape, Depth cues

Keywords: Shape Perception, Shape Representation, Texture, Principal Directions

\section{Introduction}

An important objective of visualization applications is to portray scientific data in such a way that the important patterns of the data, which may not be readily apparent in its raw format, can be accurately and intuitively communicated. Through appropriate representations of the data, visualization can speed up the cognitive process of extracting relevant information and help users make critical decisions.

Research has shown that human observers are not able to reliably perceive local shapes correctly with shading alone, without other shape cues. Studies have suggested that texture can be a visual cue to three dimensional structure of objects. Scientific study of texture as a cue in visual perception began with Gibson [Gibson 1950].

\footnotetext{
*e-mail: skim@cs.umn.edu

†e-mail:haleh@cs.umn.edu

†e-mail:interran@cs.umn.edu
}

Gibson suggested that the perception of the surface slant depends on the rate of change in the projective distortion of surface texture. Witkin [Witkin 1981] noted that the foreshortening effect (the fact the image of a slated pattern is systematically compressed in the direction of surface slat) can be used as a cue to surface orientation. Recent findings support the idea that the facility with which we can accurately perceive surface shape in the presence of surface texture depends not only upon texture characteristics but also upon how the pattern is laid down over the surface ( [Todd and Reichel 1990; Stevens 1983; Mamassian and Landy 1998; Li and Zaidi 2000; Li and Zaidi 2001; Zaidi and Li 2002; Knill 2001]).

In our own previous work, published elsewhere [Interrante and Kim 2001], we measured the accuracy of observers' estimates of surface normal direction in terms of the deviation in 3D angle from the ground-truth surface normal direction. Observers were presented with a local image of a surface textured with one of four texture patterns (principal, uniform, swirly, and isotropic 1) and asked to adjust an array of 49 probes [Koenderink et al. 1992] that completely cover the central area of the surface. Observers adjusted each probe until they were satisfied that the perpendicular extension of the probe was aligned with the surface normal direction. The study found that under flat viewing condition performance was significantly better with the isotropic pattern and the principal direction oriented pattern. Under stereo viewing accuracy increased for all textures, but errors were still slightly higher for the uniform and swirly patterns.

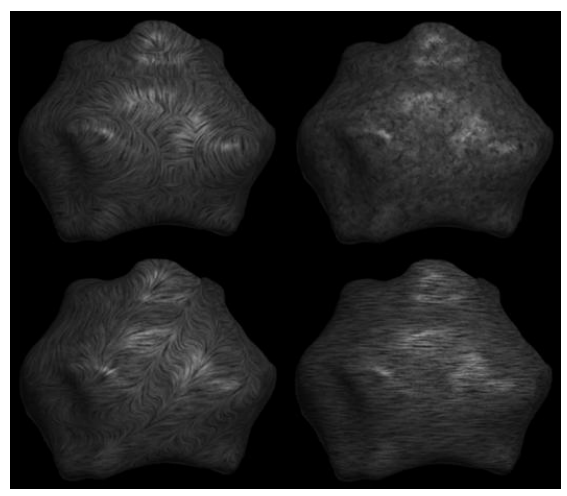

Figure 1: Example of the 3D textured surfaces in previous and present studies. Clockwise from top left: Principal, Isotropic, Swirly, and Uniform direction.

In a follow-up experiment, we included both surface relief and luminance textures. We hypothesized that: 1) the pattern of errors would be similar; and that 2) the error would be smaller under relief texture conditions than under luminance texture conditions. We expected that the relief texture patterns that follow swirly or uniform directions would not hide the underlying surface shape as much as the luminance textures would. And therefore, relief texture patterns would better perform than luminance textures. The results from the replicated experiment confirmed the first hypothesis but there was not enough evidence to support the second hypothesis.

We speculate that this might have been because the length of the experiment was too long resulting in decreased performance in the later trials. Our decision to lay the probes in a $7 \times 7$ equally-spaced 
grid might also have interfered with subjects' ability to perceive the probes as lying on the surface. Also, because some parts of the surfaces were near fronto-parallel, it was difficult to know whether a probe was untouched because the subjects perceived that location to be fronto-paralle or because they accidentally missed the probe. Finally,we speculate that not all participants had completely understood the task and a better training is needed. In the present study, we decided to focus on the difference between the two different texture mapping methods.

\section{Methods}

\subsection{Stimuli}

To limit the complexity of the task in this study, we first chose four surfaces from the original six surfaces used in earlier studies. From each of the four chosen surfaces, we randomly chose 2 probe locations of the original 49 probes. The only condition we imposed for the probe locations was that the surface normal at that point is at least $20^{\circ}$ or greater off from the initial probe direction of $\{0,0,1\}$.

There were a total of 640 trials for the study:

- 4 surfacesx

- 4 texture orientation types (principal, swirly, uniform, and isotropic)

- 2 texture mapping methods (luminance and surface relief)

- 2 probe locations (per surface)

- 2 viewing conditions (stereo and flat)

- 5 repeated measures

The stimuli were cropped images of the front-facing portions of textured level surfaces rendered in perspective projection. The images were rendered with a hybrid renderer [Interrante et al. 1997] which uses raycasting [Levoy 1988] with a Marching Cubes algorithm [Lorensen and Cline 1988] for surface localization. We used a high-quality three-dimensional line integral convolution algorithm [Stalling and Hege 1995] to define solid textures on the surfaces. The textures followed four different vector fields to define four different texture orientation conditions:

- principal (follows first principal direction)

- uniform (follows constant uniform direction on the surface)

- swirly (follows non-geodesic paths unrelated to surface geometry)

- isotropic

Figure 2 shows the stimuli used in this experiment. For the stereo trials, two images, one for each eye, were generated for each trial and subjects wore stereo eyeglasses. The complete stimuli can be seen in the color plate.

\subsection{Observers}

Five male observers participated in this study. One was a highschool student and the others were undergraduate and graduate students in Computer Science. All subjects were naïve to the purposes of the experiment and had normal or corrected-to-normal visual acuity.

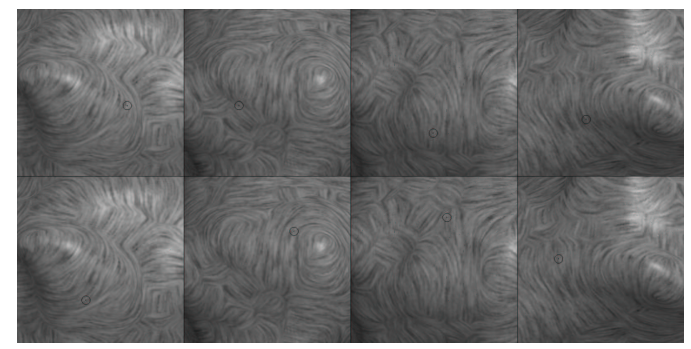

(a) luminance textures

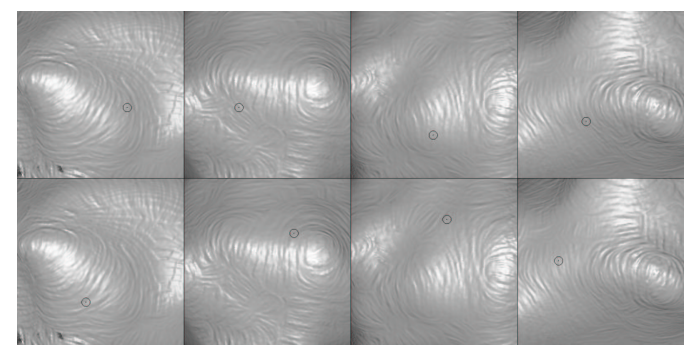

(b) surface relief textures

Figure 2: The four surfaces used in this study with principal direction. For each surface, two probe positions were chosen. Top row displays probes $0,2,4,8$; bottom row displays probes $1,3,5,7$.

\subsection{Procedure}

The experiment consisted of two sessions. In the first session subjects saw stimuli in binocular flat condition and in the second session subjects saw the same stimuli in stereo. The sessions were conducted at least 24 hours apart to ensure that subjects rest before each session and also to minimize learning effects. Each session was further divided into two sets of trials: luminance and relief. Subjects first saw surfaces with luminance textures and then with relief textures.

The experiment was run on an SGI Onyx workstation and the stimuli were displayed on a 21 inch Silicon Graphics monitor. Observers were asked to manipulate a single probe in each trial until they felt that the circular base of the probe lies flat on the tangent plane of the surface (or the perpendicular extension of the probe points to the same direction as the surface normal). We collected the surface normal vector indicated by the subjects and computed the angle in $\mathfrak{R}^{3}$ between the estimated normal direction and the true surface normal at the probe center. No feedback was given during the experiment and subjects were allowed as much time as they needed.

\subsection{Training}

Prior to the experiment, subjects were required to complete a training session in which they were asked to manipulate 15 probes, one at a time, on a surface not included in the test data. Subjects were asked to adjust a probe until they were satisfied that the perpendicular extension of the probe was aligned with the surface normal at the probe center. If the surface normal indicated by the probe was within 10 degrees of the true surface normal, they would be allowed 
to proceed to the next trial. Otherwise, the probe would be colorcoded based on the magnitude of the error. At this point, the users could continue to adjust the probe assisted by the color as a cue to correct their estimate. Subjects had to pass three out of the 15 trials without the color cues before proceeding to the actual experiment.

\subsection{Findings}
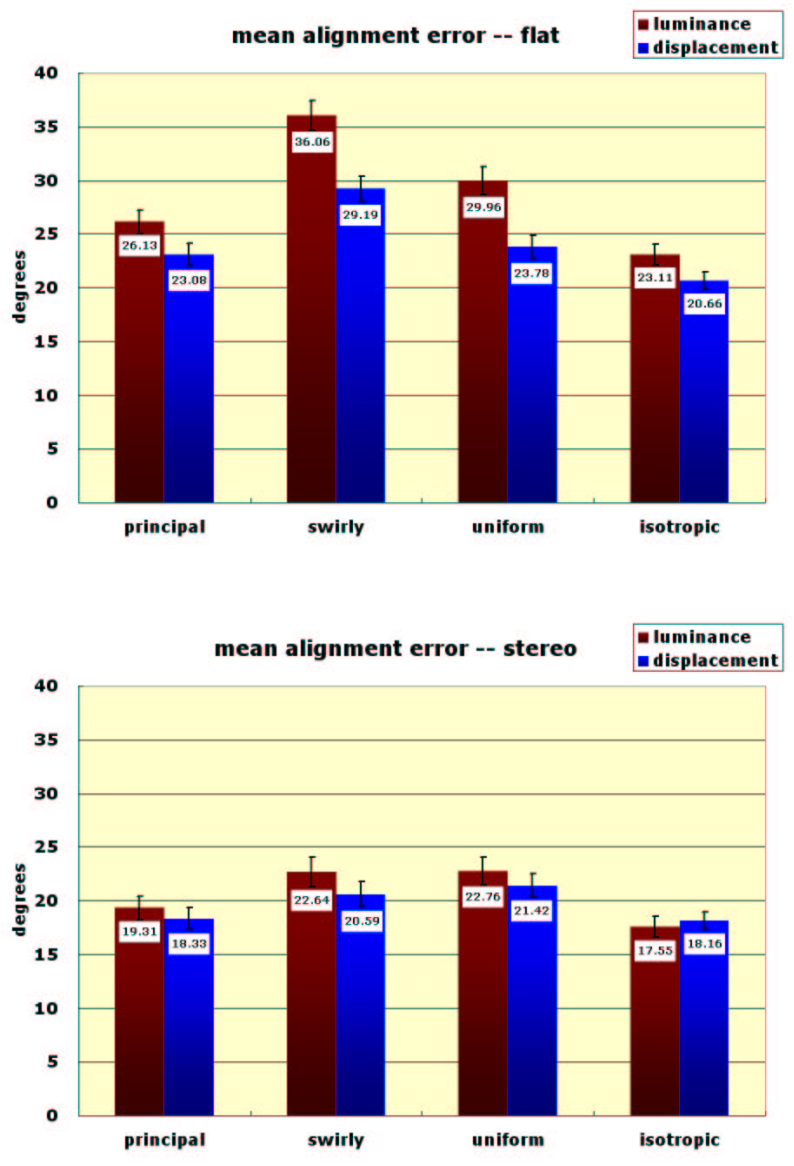

Figure 3: Pooled results (mean angular error with 95\% confidence interval) for all subjects, all surfaces, by texture type.

Figure 3 shows an overall summary of the results we found in this study. The results confirm both our hypotheses and also our findings from Experiment 1.

Figure 3 (a) shows the mean angular error and 95\% confidence interval computed over the 8 probe estimates $x 5$ repeated measures under conditions of binocular flat viewing. The results are grouped by texture type, and then by subject. The mean alignment error across luminance and relief texture mapping methods had the same pattern. Under the binocular flat viewing condition, performance was best in the case of isotropic pattern, followed by principal oriented pattern, and then by uniform oriented pattern. Performance was worst in the case of swirly pattern (sinusoidally varying directions on the surface). The results confirmed our second hypothesis that performance would be significantly better with relief textures than with luminance textures.

Figure 3 (b) shows the results under conditions of stereo viewing.
Under the stereo viewing condition, the mean alignment error was smallest in the case of isotropic pattern, closely followed by principal direction pattern, then by swirly pattern and uniform pattern. We did not find statistical significance between isotropic and principal patterns, nor between swirly and uniform patterns. Displacement texture mapping was not significantly better than luminance texture mapping. Performance was significantly better for all texture types than under flat viewing condition.

We performed a 5-way within-subject analysis of variance (ANOVA) on the overall results and looked at the 3D angle error as a function of subject $\mathrm{x}$ texture type $\mathrm{x}$ probe position $\mathrm{x}$ texture mapping $\mathrm{x}$ viewing conditions. We found significant main effects for subject $(p=1.94 e-13)$, texture type $(p=0.001242)$, probe position $(p=0.0001685)$, and viewing conditions $(p=0.02744)$. Texture mapping was marginally significant $(p=0.07063)$. We speculate that under the stereo condition texture mapping does not make much difference and this affected the overall analysis. Table 1 shows the results of ANOVA.

We used Tukey's HSD ('Honestly Significant Difference') method for more detailed analysis into texture type, mapping, and view variables. In $95 \%$ significance level, we found that the following differences were statistically significant: isotropic $<$ principal $<$ uniform $<$ swirly, stereo $<$ flat, and relief $<$ luminance.

\begin{tabular}{|l|c|r|r|}
\hline variable & DF & $F$ & $p$ value \\
\hline subject & 4 & 138.3 & $1.94 \mathrm{e}-13$ \\
\hline texture mapping & 1 & 5.99 & 0.07063 \\
\hline texture type & 3 & 10.26 & 0.001242 \\
\hline probe id & 7 & 6.311 & 0.0001685 \\
\hline viewing & 1 & 11.52 & 0.02744 \\
\hline
\end{tabular}

Table 1: Anova summary for overall results, pooled over all subjects, texture orientations, texture mapping methods, probe locations, and viewing conditions

Given that viewing condition is a significant main effect, to further study the effects of texture type and mapping methods, we divided the results into two sets - flat and stereo. The result of ANOVA is summarized in Table 2. Under the flat viewing condition, subject, texture orientation, probe location and texture mapping methods were all significant main effects. Tukey's HSD analysis revealed the same pattern as the overall results.

\begin{tabular}{|l|c|r|r|}
\hline variable & DF & $F$ & $p$ value \\
\hline texture mapping & 1 & 12.49 & 0.02414 \\
\hline texture type & 3 & 17.55 & 0.0001099 \\
\hline probe id & 7 & 6.367 & 0.0001574 \\
\hline
\end{tabular}

Table 2: Anova summary for results under flat viewing.

\begin{tabular}{|l|c|r|r|}
\hline variable & DF & $F$ & $p$ value \\
\hline texture mapping & 1 & 0.299 & 0.6136 \\
\hline texture type & 3 & 2.083 & 0.156 \\
\hline probe id & 7 & 4.216 & 0.002749 \\
\hline
\end{tabular}

Table 3: Anova summary for results under stereo viewing.

Under the stereo viewing condition, the results of ANOVA (see Table 3) summarize that neither texture type nor texture mapping methods are significant main effects. Tukey's HSD analysis found that at the 0.05 level performance was significantly better with isotropic and principal direction patterns than with swirly and uniform textures.

In order to gain deeper insight into the details of the effects of texture on surface curvature estimate, we looked separately at the re- 
sults for the luminance-flat, relief-flat, luminance-stereo, and reliefstereo stimuli. The results of Anova on each set are summarized in Tables 4 and 5 .

\begin{tabular}{|l||c|r|r||r|r|r|}
\hline \multicolumn{1}{|c||}{} & \multicolumn{3}{|c||}{ luminance-flat } & \multicolumn{3}{c|}{ relief-flat } \\
\hline variable & DF & $F$ & $p$ value & DF & $F$ & $p$ value \\
\hline texture type & 3 & 15.13 & 0.0002221 & 3 & 6.669 & 0.006706 \\
\hline probe id & 7 & 5.192 & 0.0007044 & 7 & 6.141 & 0.0002076 \\
\hline
\end{tabular}

Table 4: Anova summary for detailed results under flat viewing.

\begin{tabular}{|l||c|r|r||r|r|r|}
\hline \multicolumn{1}{|c||}{} & \multicolumn{3}{c||}{ luminance-stereo } & \multicolumn{3}{c|}{ relief-stereo } \\
\hline variable & DF & $F$ & $p$ value & DF & $F$ & $p$ value \\
\hline texture type & 3 & 3.229 & 0.0609 & 3 & 0.4656 & 0.7116 \\
\hline probe id & 7 & 2.998 & 0.01764 & 7 & 4.353 & 0.002253 \\
\hline
\end{tabular}

Table 5: Anova summary for detailed results under stereo viewing.

\begin{tabular}{|l|l|}
\hline \multicolumn{1}{|c|}{ condition } & \multicolumn{1}{|c|}{ HSD result $^{1}$} \\
\hline luminance-flat & (isotropic $<$ principal $)<$ uniform $<$ swirly \\
\hline relief-flat & (isotropic $<$ uniform $<$ principal $)<$ swirly \\
\hline luminance-stereo & (isotropic $<$ principal $)<($ swirly $<$ uniform $)$ \\
\hline relief-stereo & (isotropic $<$ principal $<$ uniform $<$ swirly) \\
\hline
\end{tabular}

Table 6: Tukey's HSD analysis summary for detailed results

\section{Conclusion}

Overall, the results from this experiment confirmed both of our hypotheses. Observers performed shape judgment task better when the texture pattern is isotropic or follows the principal direction than the other anisotropic textures which either followed a constant uniform direction on the object space or sinusoidally varying non-geodesic paths. Performance significantly improved when the surfaces were rendered with relief textures than with luminance textures under the flat viewing condition and the improvement was marginally significant under the stereo viewing condition. The surface normal estimate was significantly more accurate in the case of stereo viewing than flat viewing. Under the stereo viewing condition, texture type and texture mapping methods were not significant main effects. Tukey's HSD analysis did not find significant difference between principal oriented texture patterns and isotropic patterns.

\section{Acknowledgement}

This work was partially supported by an NSF Presidential Early Career Award for Scientists and Engineers (PECASE). The radiation dose data used in this experiment were provided by Dr. Julian Rosenman and the images were rendered using parts of volume rendering software written originally by Marc Levoy. We are grateful to our observers for their dedicated and conscientious efforts.

\footnotetext{
${ }^{1} a<b$ : HSD found significant difference between effects $a$ and $b$ at 0.05 level, performance is better in the case of $a$ than $b$ (error was smaller with $a$ than with $b) ;(a<b)$ : the difference is not significant at 0.05 level
}

\section{References}

Erens, R., Kappers, A., And Koenderink, J. 1993. Perception of local shape from shading. Perception \& Psychophysics $54,2,145-156$.

GiBson, J. 1950. The perception of visual surfaces. American Journal of Psychology 63, 367 - 384.

InTERRANTE, V., AND KIM, S. 2001. Investigating the effect of texture orientation on shape perception. In Proceedings of Human Vision and Electronic Imaging VI, SPIE 4200, 330 - 339.

Interrante, V., Fuchs, H., And PIZER, S. 1997. Conveying the $3 \mathrm{~d}$ shape of smoothly curving transparent surfaces via texture. IEEE Computer Graphics and Applications 3, 2, 98 - 117.

KNILL, D. 2001. Contour into texture: The information content of surface contours and texture flow. Journal of the Optical Society of America, A 18, 1, 12 - 35 .

Koenderink, J., van Doorn, A., And Kappers, A. 1992. Surface perception in pictures. Perception 52, 487 - 496.

LEvoy, M. 1988. Display of surfaces in volume data. IEEE Computer Graphics and Applications 8, 3, 29 - 37.

LI, A., AND ZAIDI, Q. 2000. Perception of three-dimensional shape from texture is based on patterns of oriented energy. Vision Research 40, 217 - 242.

LI, A., AND ZAIDI, Q. 2001. Information limitations in perception of shape from texture. Vision Research 41, 1519 - 1534.

Lorensen, W., And Cline, H. 1988. Marching cubes: A high resolution 3d surface construction algorithm. In Proceedings of ACM Conference on Computer Graphics and Interactive Techniques, 163 - 169 .

Mamassian, P., And Kersten, D. 1996. Illumination, shading and perception of local orientation. Vision Research 36, 15, 2351 -2367 .

Mamassian, P., AND LANDY, M. 1998. Observer biases in the 3d interpretation of line drawings. Vision Research 38, 2817 2832.

Stalling, D., And Hege, H.-C. 1995. Fast and resolution independent line integral convolution. In Proceedings of ACM Conference on Computer Graphics and Interactive Techniques, 249 -256 .

SteVens, K. 1983. The line of curvature constraint and the interpretation of $3 \mathrm{~d}$ shape from parallel surface contours. In Proceedings of the International Joint Conference on Artificial Intelligence, 1057 - 1061

TODD, J., AND REICHEL, F. 1990. Visual perception of smoothly curved surfaces from double-projected contour patterns. Journal of Experimental Psychology: Human Perception and Performance 16, 3, $665-674$.

WiTKIN, A. 1981. Recovering surface shape and orientation from texture. Journal of Artificial Intelligence 17, 17 - 45.

ZAIDI, Q., AND LI, A. 2002. Limitations on shape information provided by texture cues. Vision Research 42, $815-835$. 


\section{Conveying Three-Dimensional Shape with Texture \\ Kim, Hagh-Shenas, Interrante}
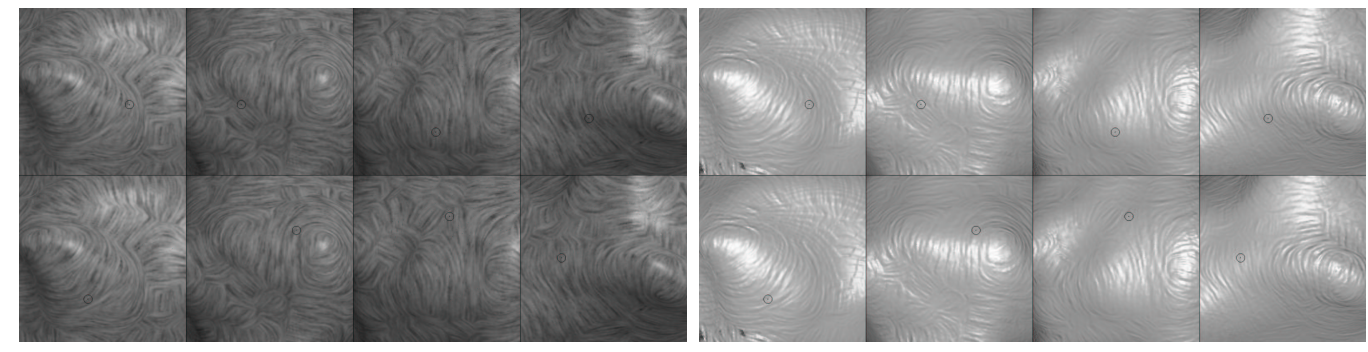

Figure 1: Principal direction stimuli used.
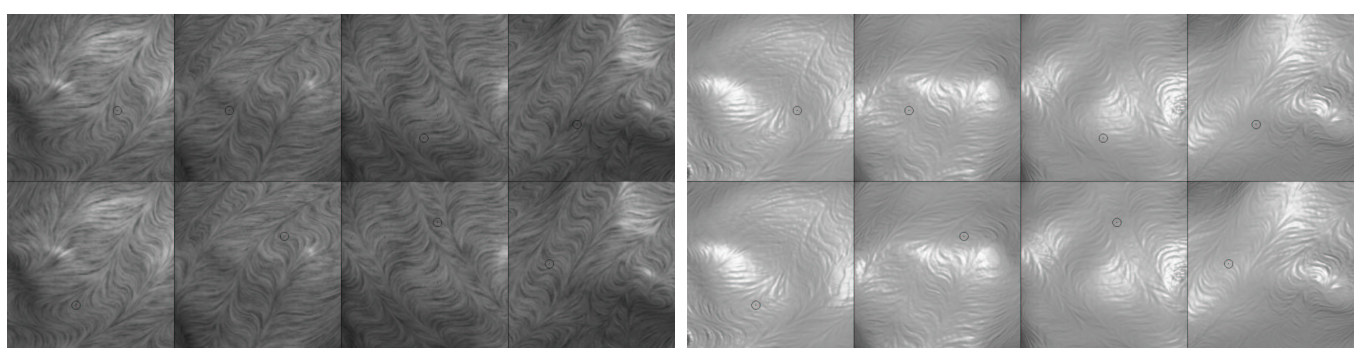

Figure 2: Swirly direction stimuli used.
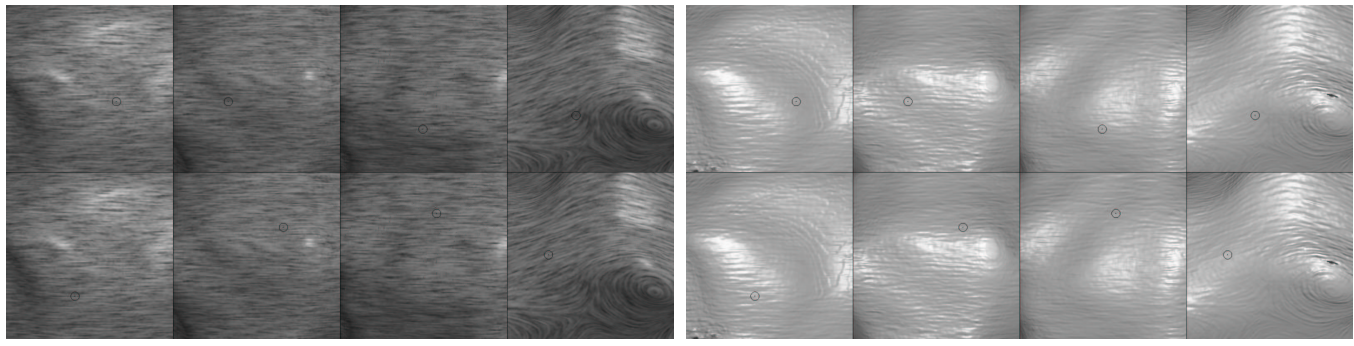

Figure 3: Uniform direction stimuli used.
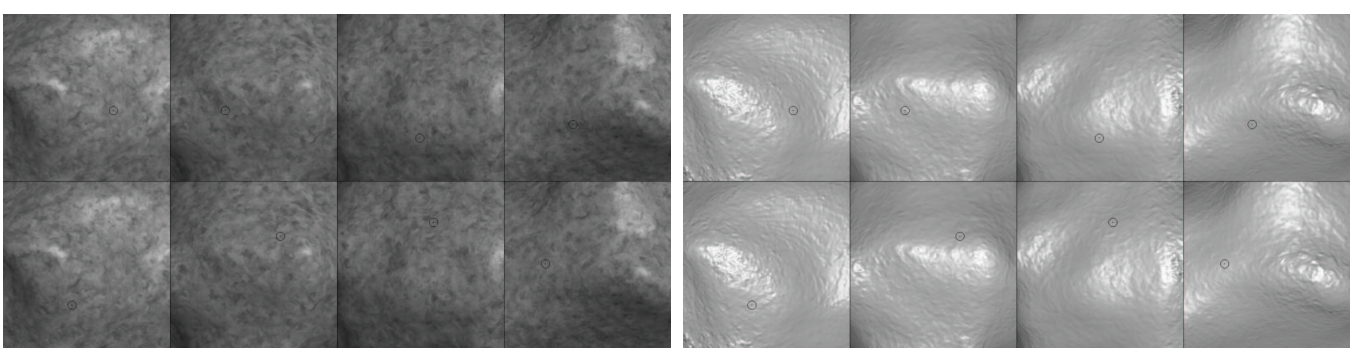

Figure 4: Isotropic stimuli used. 